\title{
Seroprevalence of Sickle Cell Anemia And Thalassemia in Suspected Case of Genetic Disorders in Tribal Predominant Population, Ranchi ,India
}

\author{
Dr.Chandrahas Prasad ${ }^{1}$,Dr. Shashi Bhushan Singh ${ }^{2}$ \\ ${ }^{1}$ Associate Professor, Department Of Laboratory Medicine, ${ }^{2}$ Associate Professor Cum Statistician, Department \\ Of Preventive And Social Medicine, Rajendra Institute Of Medical Sciences, Ranchi
}

\begin{abstract}
Introduction: The Hemoglobinopathies are one of the major public health problems in tribal predominant population.

Objective: To estimate the seroprevalence of Sickle Cell Anemia and Thalassemia in suspected cases of genetic disorders by using HPLC retention time chromatogram method in tribal predominant population, Ranchi, Jharkhand.

Methods: All Blood sample of suspected cases of genetic disorders were assessed in the department of Laboratory Medicine, Rajendra Institute of Medical Sciences, Ranchi, India for work up of anemia or other blood related disorders in year 2013. This blood samples were assessed on BIORAD variant II.

Results: A total of 107 blood samples were examined by HPLC retention time chromatogram. Out of these, 63(58.88\%) cases showed abnormal hemoglobin fractions. The major abnormality observed was of high HbA2 . A cutoff value of $>3.9 \%$ was considered for diagnosis of beta thalassemia trait (BTT). A total of 13 cases (12.1\%) of BTT were diagnosed. Other hemoglobinopathies were as follows: Sickle homo 12 (11.2\%), Sickle trait 10 (9.3\%), dB Thal trait 9 (8.4\%), Sickle Thal 7 (6.5\%), Heredetery Persistent Fetal Hemoglobin (HPFH) $7(6.5 \%)$, B Thal Major 5 (4.6\%).

Conclusion: This study revealed that seroprevalence of Sickle Cell Anemia and Thalassemia is $58.88 \%$ in study sample.
\end{abstract}

Keywords: Retention time chromatogram , HPLC, Hemoglobinopathies, Thalassemia, Sickle cell Anemia.

\section{Introduction}

Haemoglobin is a complex molecule contained within erythrocytes that binds to and transports oxygen and carbon dioxide in the body. Normal alpha 2 beta 2 chain genes for globin chain synthesis are located in two clusters on chromosome $11 \& 16$. Genetic mutation causes reduced synthesis of beta chain to ineffective erythropoesis causing reduced hemoglobin. Defects in genes of haemoglobin can produce abnormal haemoglobins and anemia, which leads to conditions, termed as "haemoglobinopathies". Abnormal haemoglobins appear in one of two basic circumstances: decreased production of one of the globin chain e.g. thalassaemia, abnormal globin chain e.g. sickle cell disease. ${ }^{1}$

Abnormalities of hemoglobin $(\mathrm{Hb})$ synthesis are among the most common inherited disorders of man and can be quantitative (thalassemia syndrome) or qualitative (variant $\mathrm{Hb} \mathrm{S})^{2}$. Of these, thalassemia syndromes particularly beta thalassemia major is serious and a major cause of morbidity. WHO figures estimate that $5 \%$ of the world population is a carrier for hemoglobin disorders ${ }^{3}$.The frequency of B-thalassemia in India ranges from 3.5 to $15 \%$ in general population ${ }^{4}$. Every year 10,000 children with thalassaemia major are born in India, which constitutes $10 \%$ of the total numbers in the world. ${ }^{5}$ India spends nearly Rs 1000 crore per annum in the treatment of thalassemia patients. Majority of the centers in India use conventional methods for diagnosis of haemoglobinopathies, which include clinical and family history, red cell indices, complete blood counts (CBC), $\mathrm{HbA} 2, \mathrm{HbF}$ estimation, sickling test and $\mathrm{Hb}$ electrophoresis. ${ }^{6}$ Electrophoresis of hemoglobin variants with similar mobilities has inherent limitations. The identification of variants is dependent on the technical performance of electrophoresis, which has many variables, e.g., hemoglobin concentration, amperage, running temperature, and length of electrophoresis run. These variables can affect the quality of separation and relative positioning of the bands. Variants that migrate identically or similarly would be very difficult, if not impossible, to evaluate without the unknown sample being electrophoresed directly adjacent to the reference hemoglobin mixture or adjacent to several known stored specimens. HPLC, on the other hand, has been shown to have a high degree of reproducibility and precision. ${ }^{18}$

Double heterozygous states between certain variants can also lead to hematological defects. The use of cation - exchange high performance liquid chromatography (CE-HPLC) to separate and quantify various normal 
and abnormal hemoglobin $(\mathrm{Hb})$ fractions has been increasing ${ }^{7}$. It offers a reliable tool for early, accurate detection thereby aiding in the prevention and management of various hemoglobinopathies ${ }^{8}$.

\section{Material \& Methods}

Blood sample of suspected cases of gentic disorders were assessed in the department of Laboratory Medicine of RIMS, Ranchi for work up of anemia or other blood related disorders. This blood samples were assessed on BIORAD variant using beta thalassemia short program. A total of 107 cases, in the year 2013 for $\mathrm{Hb}$ variant analysis were studied for various hemoglobinopathies and variants. 107 blood samples in the year 2013, were run in 5 parts SYSMAX hemato- analyzer before performing HPLC to obtain the Hemoglobin values and indices. The tests were performed on BIO RAD 'VARIANT II which utilizes the principle of high performance liquid chromatography (HPLC). An Hb A2/F calibrator and two levels of controls (BIO-RAD) were analyzed at the beginning of each run. The total area acceptable was between-one to three million. History of Blood transfusion and relevant family history was taken in all cases. The software delivers a printed report showing the chromatogram, with all the hemoglobin fractions eluted. The integrated peaks are assigned to the manufacturer - defined "windows" derived from the specific retention time (RT). This retention time is the time that elapses from the sample injection to the apex of the elution peak, of normal hemoglobin fraction and common variants (Table 1). The "windows" are established ranges in which common variants have been observed. The printed chromatogram shows all the hemoglobin fractions eluted, the retention times, the areas of the peaks and the values (\%) of different hemoglobin components. If a peak elutes at a retention time that is not predefined, it is labelled as an unknown. Each analytical cycle, from sampling to printing of results takes about only a few minutes ( about 6.5 minutes). ${ }^{9}$

Alkaline gel electrophoretic tests was also done in few selected cases just to compare the results.

Table-1Manufacturer assigned windows for BIO Rad Variant II HPLC system ${ }^{9}$.

Analysis of Hbs by HPLC

Table 1

Analyte Identification Windowa

\begin{tabular}{lccc}
\hline Analyte name & Retention time $(\mathrm{min})$ & Band $(\mathrm{min})$ & Window $(\mathrm{min})$ \\
\hline F & 1.15 & 0.15 & $1.00-1.30$ \\
P2 & 1.45 & 0.15 & $1.30-1.60$ \\
P3 & 1.75 & 0.15 & $1.50-1.90$ \\
A0 & 2.60 & 0.40 & $2.20-3.30$ \\
A2 & 3.83 & 0.15 & $3.68-3.98$ \\
D-window & 4.05 & 0.07 & $3.98-4.12$ \\
S-window & 4.27 & 0.15 & $4.12-4.42$ \\
C-window & 5.03 & 0.15 & $4.88-5.18$ \\
\hline
\end{tabular}

${ }^{a}$ Example provided by manufacturer.

23

\section{Results}

Laboratory records of 107 patients of anemia with suspected hemoglobinopathies were analyzed and $63(67.40 \% \%)$ patients showed different abnormal hemoglobin variants (table 2). Out of total 107 cases, 71 ( $75.97 \%$ ) were male and 36 ( $38.52 \%$ ) were female. Criteria for suspecting hemoglobinopathy in these cases included: results of screening tests eg., various discriminant functions (so obtained on hematology cell counters, findings obtained from the peripheral smear examination, family history and relevant clinical signs and symptoms suggestive of hemoglobinopathy.44 (47.08\%) cases which were labeled as "NoHemoglobinopathies" also included cases of Iron Deficiency anemia \& Malaria amongst the other ones having other causes of anemia.The age range of patients was from 1 month to 18 years. Of these $13(21.1 \%)$ patients were diagnosed to have beta-heterozygous thalassaemia (Table 2) based on the high level of HbA2 (>3.9\%). These patients presented with mild anaemia (mean $\mathrm{Hb}$ level of $10.4 \mathrm{gm} / \mathrm{dl}$ ) and all showed microcytic hypochromic blood picture with low mean corpuscular volume RDWSD < $46 \mathrm{fl}$, RDW CV < $16 \%$ [(MCV) of $<80 \mathrm{fl}$ ]. $5(4.6 \%)$ of 107 cases were diagnosed as beta - homozygous thalassemia (Table 2). All these patients had increased $\mathrm{HbF}$ values $(>40 \%)$. Clinically they presented with severe pallor requiring regular blood transfusion and had moderate to marked hepatosplenomegaly. $9(8.4 \%)$ cases of d B Thal Trait [ [ Hb A2 ( $4-$ $7 \%) \&(7-9 \%)]$ were diagnosed \& $7(6.5 \%)$ of HPFH Trait ( Hb F $15-40 \%$ ) along with variable degree of anemia showing anisopoikilocytosis and microcytic hypochromic (MCHC) blood picture. Hb F levels were raised with a variable reduction in $\mathrm{Hb} \mathrm{A}$. and these patients were not dependent on blood transfusion. 12 (11.2 
$\%)$ and $10(9.3 \%)$ patients were diagnosed as sickle cell anemia (Table 2) and sickle cell trait respectively. The diagnosis was reconfirmed by positive cycling test.7 ( $6.5 \%)$ patients were diagnosed as "compound heterozygous sickle - $\beta+$ thalassemia" based on HPLC reports of the cases as well as family studies (one of the parents having $\mathrm{HbS}$ trait and the other one having BTT). HbF levels in all these cases ranged form > $2 \%$ (5$15 \%$ ), A $2>5 \%$ and $\mathrm{Hb} \mathrm{S}>50 \%$. Only one patient (1.07\%) had $\mathrm{Hb} \mathrm{J}$ Trait in which P3 was elevated $>15.1$ $\%$ ( included among others).

Table 2

\begin{tabular}{|c|c|c|}
\hline \multicolumn{3}{|c|}{$\begin{array}{l}\text { Spectrum of Thalassemia \& Sickle Cell Diseases recently } \\
\text { observed in Department of Laboratory Medicine, RIMS, Ranchi }\end{array}$} \\
\hline Type & Total & Percent \\
\hline B Thal Trait & 13 & $12.1 \%$ \\
\hline d B Thal Trait & 9 & $8.4 \%$ \\
\hline Sickle Homo & 12 & $11.2 \%$ \\
\hline Sickle Thal & 7 & $6.5 \%$ \\
\hline HPFH Trait & 7 & $6.5 \%$ \\
\hline Sickle Trait & 10 & $9.3 \%$ \\
\hline B Thal Major & 5 & $4.6 \%$ \\
\hline IDA & 21 & $19.6 \%$ \\
\hline MP & 18 & $16.8 \%$ \\
\hline Others & 5 & 4.6 \\
\hline Total & 107 & $99.6 \%$ \\
\hline
\end{tabular}

\section{Spectrum of Thalassemia \& Sickle cell anemia}

- Series1

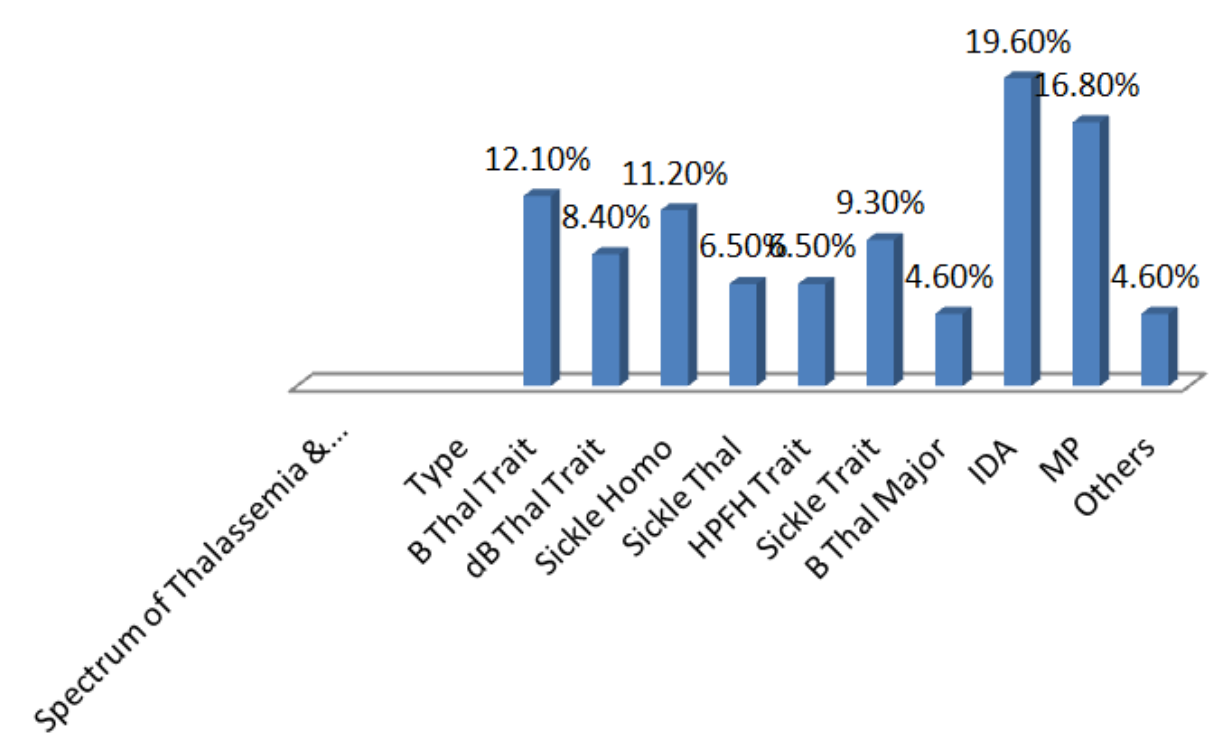



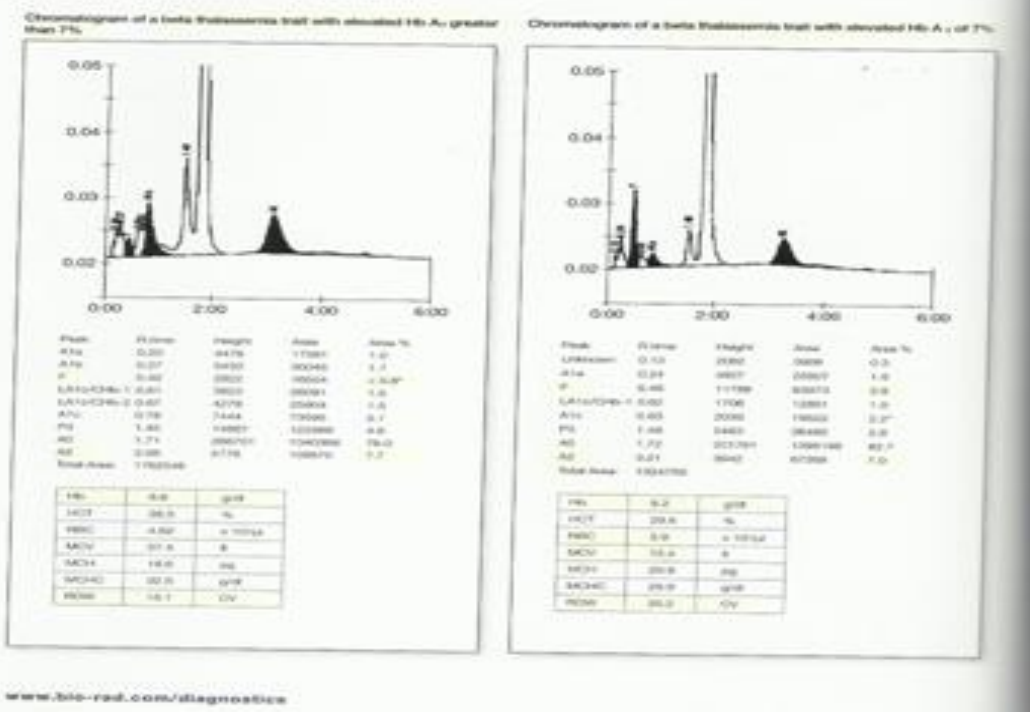

Figure 1 : Chromatogram of
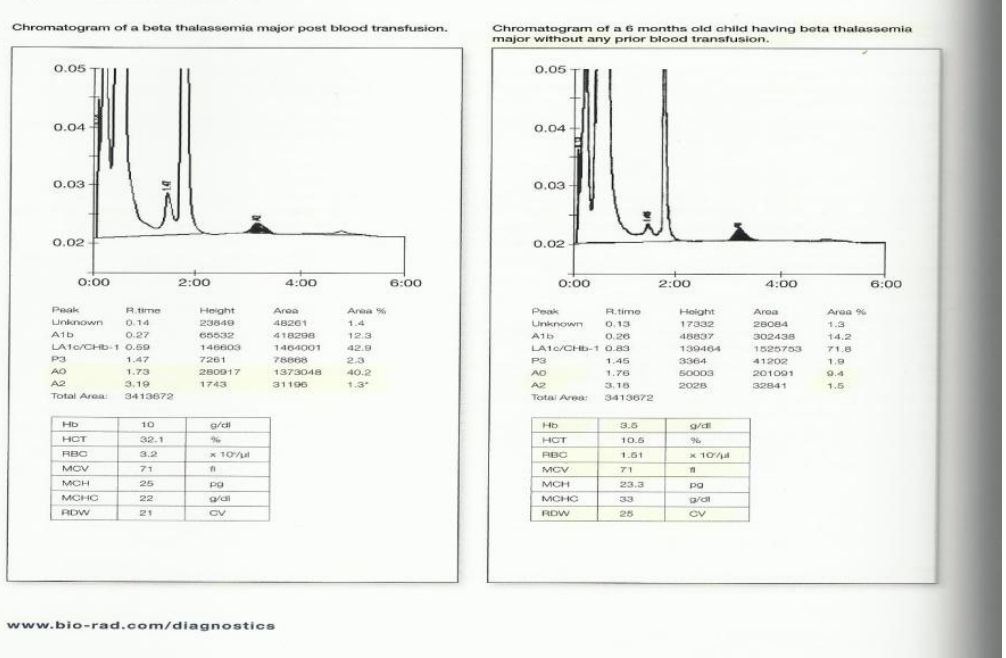

Figure 2: Chromatogram of Beta thalassemia major
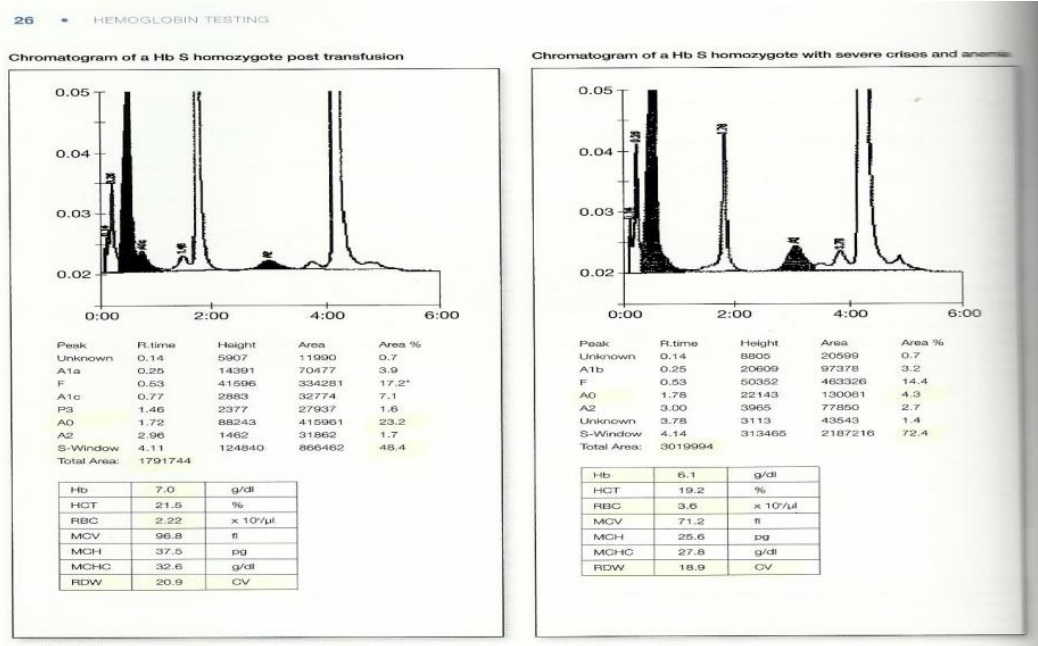

Figure 3: Chromatogram of Sickle cell anemia 

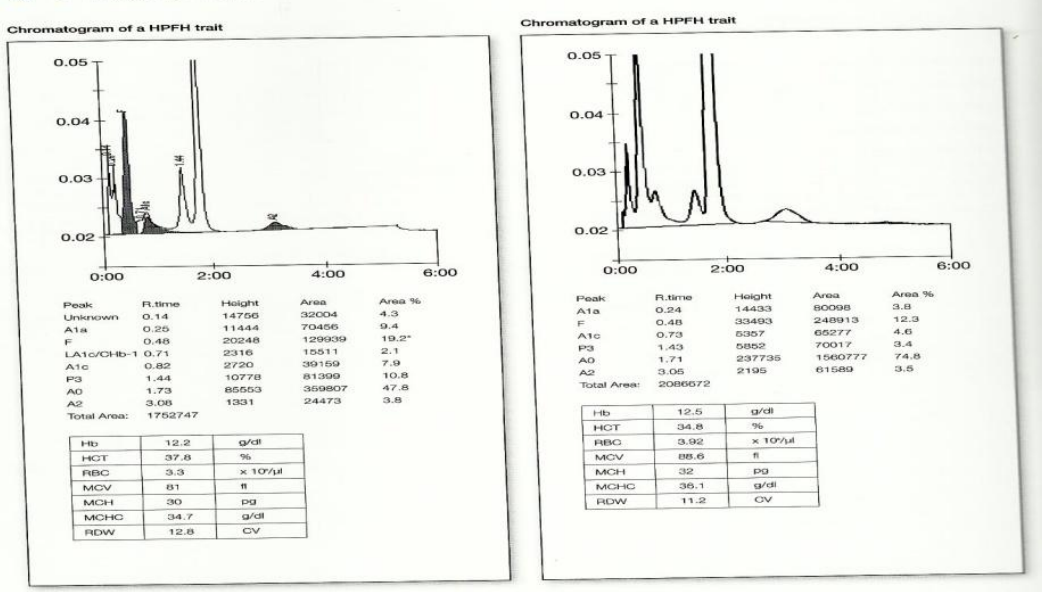

Figure 4 : Chromatogram of HPFH heterozygous

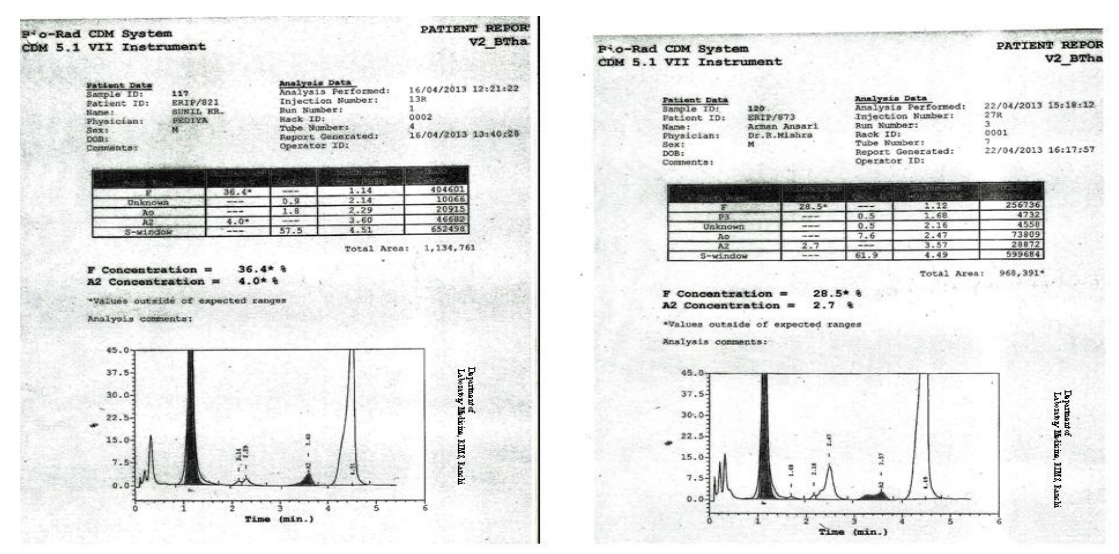

Figure - 5 Chromatogram of our study

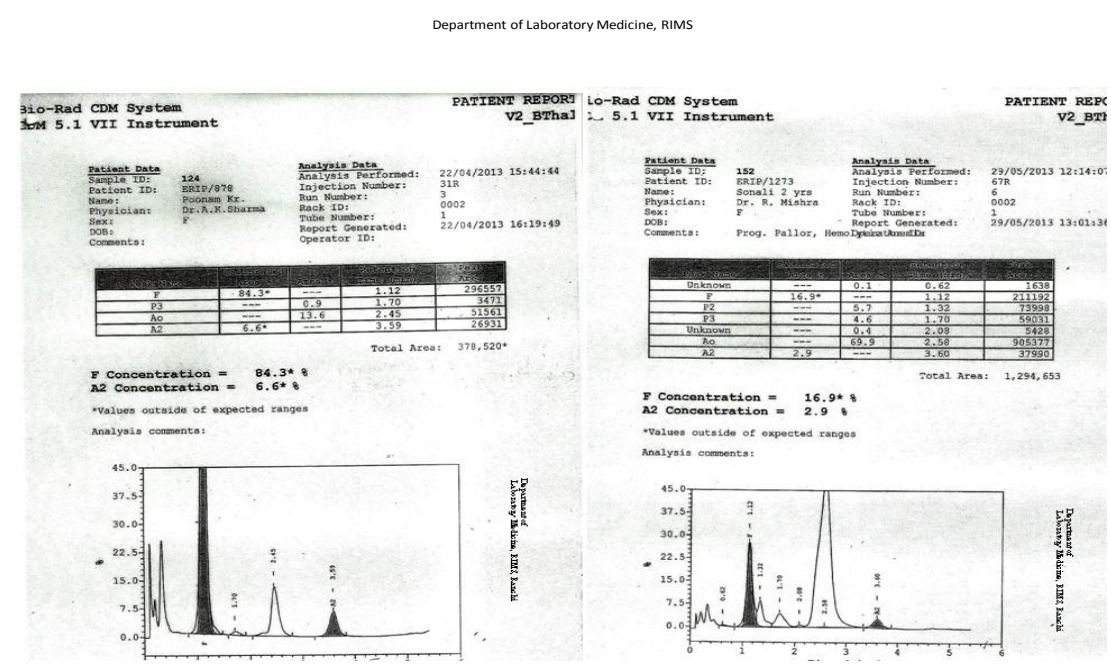

Figure - 6 Chromatogram of our study 


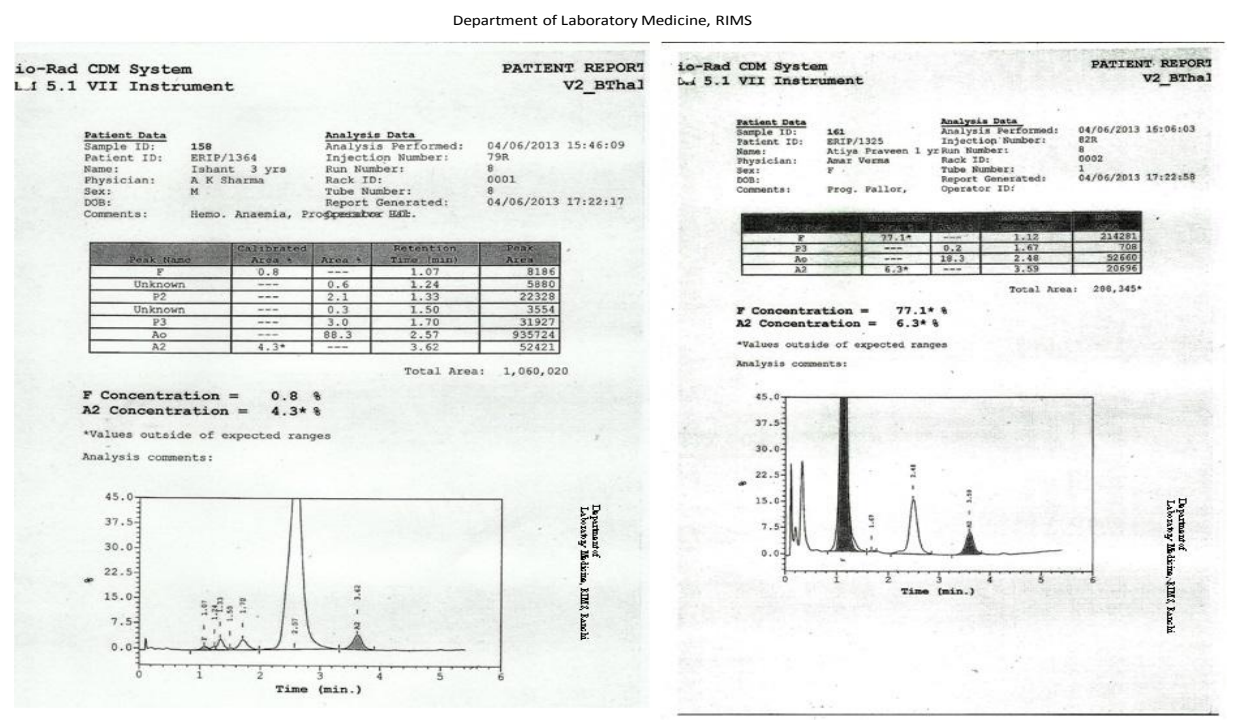

Figure - 7 Chromatogram of our study

\section{Spectrum of Thalassemia \& Sickle cell anemia}

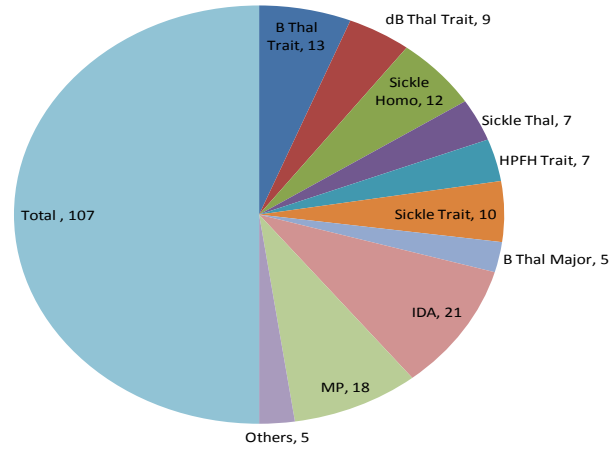

Figure - 8 Spectrum of hemoglobinopathies in our study

\section{Discussion}

Hemoglobinopathies and thalassaemias are common disorders in our country. Appropriate modernized ,well equipped, updated laboratory tests are required for diagnosis and confirmation of these disorders.

The most common investigational tools for diagnosis of hemoglobinopathies and thalassemias are alkaline and acid gel electrophoresis, $\mathrm{HbA} 2$ quantification by ion exchange column chromatography and $\mathrm{HbF}$ quantification by alkali denaturation method.

The identification of hemoglobin variants by conventional Electrophoretic techniques are often presumptive, based on the electrophoretic mobility of the band, quantification and / or ethnic origin of the parents ${ }^{6}$.

Ordinary manual alkaline agar gel electrophoresis have got certain drawbacks like 1) Procedure is very long \& cumbersome. It requires a very high speed (rpm) centrifuge. 2) Quantification of the results cannot be elucidated in the absence of a densinometer or other necessary accessories / equipment. 3) If there are two similar buffers, possibilities of mistakes . 4) Usually runs are not very clear. Bands are overlapping in different circles. 6) Ultimately conclusive results cannot be made. 7) Usually single sample facility is not there. 8) In the absence of full \& extra team of paramedical staffs of the laboratory it will generate unnecessary load of burden to the smooth lab working environment. 
The compound heterozygous disorders or unusual variants are all clinically significant with varying degree of severity, making precise identification important ${ }^{11,12}$, none can be conclusively identified by a single electrophoretic technique ${ }^{13}$.

HPLC offers the distinct advantage over classic hemoglobin electrophoresis as it can more accurately identify and quantitate abnormal haemoglobins ${ }^{14}$. It is also very useful for pediatric group of patients, as only $5 \mu \mathrm{l}$ of blood is sufficient for analysis. HPLC has been shown to be rapid, sensitive, specific and reproducible alternative to conventional hemoglobin electrophoresis.

Our study included predominantly patients of Ranchi district. It also includes a few cases from other districts of Jharkhand as RIMS being a Tertiary Care Institute. Out of 107 cases studied, 63 cases $(67.40 \%)$ displayed various abnormal hemoglobin fractions on HPLC runs. Beta thalassemia trait formed the largest subgroup of abnormal hemoglobin (12.1\%). The high incidence of Beta thalassemia trait underscores the need for both the population screening of marriageable age groups ( age of 18) and antenatal screening for prevention of thalassemia major in offspring. Thalassemia major, d B Thal Trait \& HPFH Trait constituted approximately $4.6 \%, 8.4 \%$ and $6.5 \%$ of cases respectively. ( Table 2 )

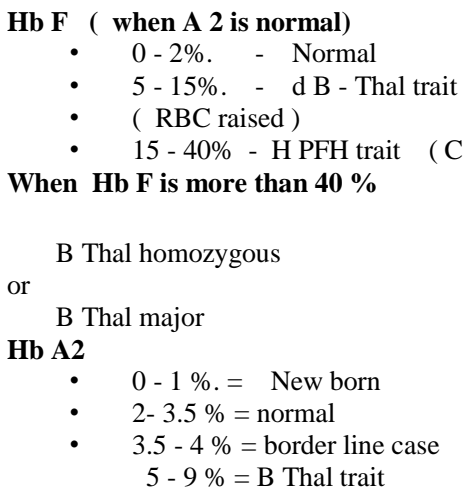

- If $\mathrm{Hb} \mathrm{S}$ more than $50 \%$ and

\section{Interpretation of HPLC results at a glance: -}

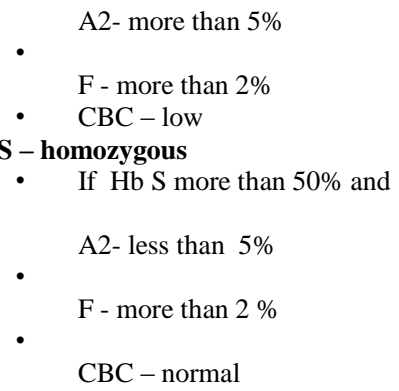

History of recent blood transfusion must be sought along with correct age so as to aid in an accurate diagnosis. Conditions with borderline $\mathrm{Hb} \mathrm{A} 2$ need careful interpretation. Iron deficiency may lead to low $\mathrm{Hb} \mathrm{A} 2$ and hence may mask a thalassemia trait where as B12/folate deficiency may lead to slightly raised $\mathrm{Hb} \mathrm{A} 2$ leading to a false diagnosis of a trait.

$\mathrm{Hb} \mathrm{S}$ homozygous (12 cases) presented with a variant $\mathrm{S}$ Window ranging from $70-90 \%$ and retention time 4.42 minutes. Sickling test was positive in all such cases. Hb was $7.2 \mathrm{gm} / \mathrm{dL}$ with target cells and few irreversibly sickled cells in the peripheral smear. Hb F was in the range of $15-20 \%$. Seventy three cases were diagnosed as $\mathrm{Hb} \mathrm{S}$ heterozygous having S- window with abnormal hemoglobin ranging from>50\%. 10 cases were diagnosed as a Sickle trait in which A2 was $<5 \%$ \& $\mathrm{Hb} \mathrm{F}<2 \%$. 7 cases of double heterozygous for $\mathrm{Hb} \mathrm{S}-$ beta thalassemia trait in which $\mathrm{Hb} \mathrm{S}>50 \%, \mathrm{~A} 2>5 \%$ \& $\mathrm{Hb} \mathrm{F}>2 \%$.

As we know that the Principle of HPLC \& Column comprises of a small cation exchange cartridge ${ }^{8}$, This makes an excellent platform for screening hemoglobin variants and hemoglobinopathies along with 
thalassemias. College of American Pathologists has shown far superiority over electrophoretic methods ( 2003 CAP survey). ${ }^{15}$

Although HbS syndromes could be diagnosed by both HPLC and gel electrophoresis, the use of HPLC helped in further sub - characterization of these syndromes based on quantification of $\mathrm{HbJ}$, $\mathrm{HbS}$ and $\mathrm{HbA} 2$ levels. In general, $\mathrm{HbJ}$ syndromes and $\mathrm{HbS}$ trait are asymptomatic but patients with sickle cell anemia (Hb SS) are symptomatic. Hb J trait results as P3 peak more than $15 \%$. elute in unknown peak window on HPLC.

It is thus recommended that HPLC should be definitely be performed for further sub characterization of rare $\mathrm{Hb}$ variants ${ }^{16}$. HPLC avoids misidentification of two hemoglobin variants having benign interaction with $\mathrm{HbS}$. With the integration of proper algorithms involving retention time, amount of $\mathrm{Hb}$ and $\mathrm{RBC}$ indices, a clinical laboratory is capable of identifying about $\mathbf{9 0} \%$ of the common variants encountered without the need for other studies such as alkaline and acid electrophoresis.

In the long run limitation of electrophoretic technique is higher capital \& reagent costs and considerable skill and experience are needed in interpreting the results, as the data produced are quite complex. 17 A disclaimer should always accompany the reports and findings must be supplemented by Hemogram findings, family/sibling studies, other confirmatory techniques and molecular studies based on HPLC findings and on a case-to-case basis.

In conclusion, the simplicity of sample preparation, accurate quantification of hemoglobin concentration combined with complete automation, makes HPLC an ideal methodology for the routine diagnosis of hemoglobin disorders. Hemoglobinopathies exert significant burden on India, especially in our state, Jharkhand. Adequate measures and screening procedures should be adopted to reduce this burden. HPLC and hemato- analyzer an excellent, powerful diagnostic tool for the direct identification of hemoglobin variants with a high degree of precision in the quantification of major and minor, normal and abnormal, hemoglobin fractions.

\section{Acknowledgement}

Author acknowledges the support of faculty of various departments \& the Director of RIMS, Ranchi. Author is giving thanks to Mr. Jawed Akhtar, senior lab tech. for rendering technical help and lab work support during the course of this study, which is gratefully acknowledged.

\section{References}

[1]. Sarnaik S. Thalassemia and related hemoglobinopathies. Indian J Pediatr 2005; 72:319-324

[2]. Kutlar F. Diagnostic approach to hemoglobinopathies. Hemoglobin 2007;31:243-50.

[3]. WHO- EXECUTIVE BOARD EB118/5, 118th Session Report by the Secretariat on Thalassaemia and other haemoglobinopathies : Prevalence of Haemoglobinopathies. 11 May 2006; p.1-8.

[4]. Balgir RS. The genetic burden of hemoglobinopathies with special reference to community health in India and the challenges ahead.Indian Journal of Hematology and Blood Transfusion. 2002; 20(1): 2-7.

[5]. Varawalla NY, Old JM, Sarkar R, Venkatean R, Weatherall DJ. The spectrum of beta thalassaemia mutations on the Indian subcontinent; the basis of prenatal diagnosis. Br J Haematol 1991;78:242-247.

[6]. Lt Col PK Gupta et al. Cation Exchange High Performance Liquid Chromatography for Diagnosis of Haemoglobinopathies. MJAFI, Vol. 65, No. 1, 2009.

[7]. Higgins TN, Ridley B. Tentative identification of hemoglobin variants in the Bio - Rad VARIANT II Hb A1C method.Clin Biochem 2005; 38: 272-7.

[8]. Riou J, Godart C, Hurtrel D, Mathis M, Bimet C, et al. Cation-exchange HPLC evaluated for presumptive identification Of hemoglobin variants Clin Chem. 1997; 43: 34-9.

[9]. Bio - Rad VARIANTIM thalassaemia short program. Instruction Manual 2003; 10

[10]. www.Biorad. Com.diagnostics.

[11]. Steinberg MH. Compound heterozygous and other sickle hemoglobinopathies. In: Steinberg MH, Forget BG, Higgs DR, Nagel RL, eds. Disorders of hemoglobin: genetics, pathophysiology, and clinical management. New York: Cambridge University Press, 2001;786-810.

[12]. Dash S. Hb A2 in subjects with Hb D. Clin Chem 1998; 44:2381-2.

[13]. Bain BJ. Haemoglobinopathy Diagnosis. Oxford: Blackwell Science Ltd 2001.

[14]. Sachdev R, Dam AR, Tyagi G. Detection of Hb variants and hemoglobinopathies in Indian population using HPLC: Report of 2600 cases. Indian J Pathol Microbiol 2010;53:57-62.

[15]. HG -C Hemoglobinopathy Participant Summary. 2003 College of American Pathologists.

[16]. Tyagi S, Saxena R, Choudhry VP. HPLC - How necessary is it for haemoglobinopathy diagnosis in India? Indian J Pathol Microbiol 2003; 46: 390-3.

[17]. Manual- protocol alkaline agar gel electrophoresis- HIMEDIA , 2011, - 3

[18]. Joutovsky A, Hadzi-Nesic J, Nardi MA. HPLC retention time as a diagnostic tool for hemoglobin variants and hemoglobinopathies: a study of 60,000 samples in a clinical diagnostic laboratory. Clin Chem 2004; 50: 1736-47. 